\title{
Superconvergence of a Collocation-Type Method for Simple Turning Points of Hammerstein Equations
}

\section{By Sunil Kumar}

\begin{abstract}
In this paper a simple turning point $\left(y=y^{c}, \lambda=\lambda^{c}\right)$ of the parameterdependent Hammerstein equation

$$
y(t)=f(t)+\lambda \int_{a}^{b} k(t, s) g(s, y(s)) d s, \quad t \in[a, b],
$$

is approximated numerically in the following way. A simple turning point $\left(z=z^{c}\right.$, $\left.\lambda=\lambda^{c}\right)$ of an equivalent equation for $z(t):=\lambda g(t, y(t))$ is computed first. This is done by solving a discretized version of a certain system of equations which has $\left(z^{c}, \lambda^{c}\right)$ as part of an isolated solution. The particular discretization used here is standard piecewise polynomial collocation. Finally, an approximation to $y^{c}$ is obtained by use of the (exact) equation

$$
y(t)=f(t)+\int_{a}^{b} k(t, s) z(s) d s, \quad t \in[a, b] .
$$

The main result of the paper is that, under suitable conditions, the approximations to $y^{c}$ and $\lambda^{c}$ are both superconvergent, that is, they both converge to their respective exact values at a faster rate than the collocation approximation (of $z^{c}$ ) does to $z^{c}$.
\end{abstract}

1. Introduction. We consider parameter-dependent Hammerstein equations of the form

$$
y(t)=f(t)+\lambda \int_{a}^{b} k(t, s) g(s, y(s)) d s, \quad t \in[a, b],
$$

where $-\infty<a<b<\infty, \lambda \in \mathbf{R}$ is the parameter, $f, k$, and $g$ are known functions, with $g(s, v)$ nonlinear in $v$, and the pair $\left(y=y^{c}, \lambda=\lambda^{c}\right)$ is a simple (that is, quadratic) turning point of (1.1) which is to be determined numerically. (For the definition of a simple turning point see the first paragraph of Section 3.)

We do this by an approximation procedure which hinges on an equivalent equation (see (1.4)) for the function $z$ defined by

$$
z(t):=\lambda g(t, y(t)), \quad t \in[a, b] .
$$

On substituting (1.2) into (1.1) we have immediately

$$
y(t)=f(t)+\int_{a}^{b} k(t, s) z(s) d s, \quad t \in[a, b],
$$

and hence it follows from (1.2) that $z$ satisfies the nonlinear integral equation

$$
z(t)=\lambda g\left(t, f(t)+\int_{a}^{b} k(t, s) z(s) d s\right), \quad t \in[a, b] .
$$

Received December 15, 1986; revised June 29, 1987.

1980 Mathematics Subject Classification (1985 Revision). Primary 65R20, 45G10. 
The equivalence of (1.4) and (1.1) (Kumar and Sloan [13]) is in the sense that there is a one-to-one correspondence between their solution sets (see Lemma 1 for details). Thus, corresponding to the simple turning point $\left(y^{c}, \lambda^{c}\right)$ of $(1.1)$ is a simple turning point $\left(z=z^{c}, \lambda=\lambda^{c}\right)$ of (1.4). It is the latter turning point that is actually computed in this paper. This computation is done via the enlarged system approach of Moore and Spence [14], with piecewise polynomial collocation (Atkinson, Graham and Sloan [4], Joe [7]) as the underlying discretization.

The desired approximation to $y^{c}$ is then obtained by use of the (exact) equation (1.3). Thus, essentially, $y^{c}$ is approximated by the collocation-type method of Kumar and Sloan [13] (see also Kumar [11]).

The rest of the paper is organized as follows. Section 2 provides necessary background material, while Section 3 gives details of our approximation procedure. Section 3 also establishes the convergence of the approximations to $z^{c}, \lambda^{c}$, and $y^{c}$, this being done there by a simple application of a theorem of Spence and Moore [16]. In Section 4 the theorem of Spence and Moore is used again, and hence it is shown that, under suitable conditions, the approximations to $y^{c}$ and $\lambda^{c}$ are both superconvergent, that is, they both converge to their respective exact values at a faster rate than the collocation approximation (of $z^{c}$ ) does to $z^{c}$. In Section 5 numerical results are presented.

2. Preliminaries. It is convenient to consider (1.1) in the Banach space $C=C[a, b]$ of continuous, real-valued functions on $[a, b]$. Recall that this space is equipped with the uniform norm

$$
\|x\|_{\infty}=\sup _{a \leq t \leq b}|x(t)|, \quad x \in C .
$$

On the other hand, it is convenient to consider (1.4) in the Banach space $R=R[a, b]$ of bounded Riemann-integrable real-valued functions on $[a, b]$, the reason being that $R$ permits piecewise continuous approximations to $z^{c}$ to be dealt with (Anselone [3, p. 22], Atkinson, Graham and Sloan [4]). Like $C$, the space $R$ is also equipped with the uniform norm. Since a bounded function on $[a, b]$ is Riemann-integrable if and only if it is continuous almost everywhere on $[a, b]$, it follows that $C$ is a closed subspace of $R$.

It is also convenient to make the following assumptions on the functions $f, k$, and $g$ in (1.1):

A1. $f \in C$;

A2. the kernel $k$ satisfies

$$
\sup _{a \leq t \leq b} \int_{a}^{b}|k(t, s)| d s<\infty
$$

and

$$
\lim _{t \rightarrow t^{\prime}} \int_{a}^{b}\left|k(t, s)-k\left(t^{\prime}, s\right)\right| d s=0, \quad t^{\prime} \in[a, b] ;
$$

A3. the function $g(t, v)$ is defined and continuous on $[a, b] \times \mathbf{R}$;

A4. the partial derivative $g_{v}(t, v):=\partial g(t, v) / \partial v$ exists and is continuous on $[a, b] \times \mathbf{R}$

A5. the partial derivative $g_{v v}(t, v):=\partial g_{v}(t, v) / \partial v$ exists and is continuous on $[a, b] \times \mathbf{R}$; 
A6. the function $g_{v v}$ satisfies the Lipschitz condition

$$
\left|g_{v v}\left(t, y_{1}(t)\right)-g_{v v}\left(t, y_{2}(t)\right)\right| \leq \alpha\left|y_{1}(t)-y_{2}(t)\right|
$$

for some constant $\alpha>0, t \in[a, b]$, and all $y_{1}, y_{2} \in B_{1}\left(y^{c}, \rho\right)$, where

$$
B_{1}\left(y^{c}, \rho\right)=\left\{y \in C:\left\|y-y^{c}\right\|_{\infty} \leq \rho\right\}, \quad \rho>0 .
$$

Note that assumption A2 ensures via the Arzelà-Ascoli theorem (Kantorovich and Akilov [9, p. 27]) that the linear integral operator $K$, defined by

$$
(K w)(t):=\int_{a}^{b} k(t, s) w(s) d s, \quad t \in[a, b], w \in R,
$$

is a compact operator from $R$ to $C$ (Anselone [1], [2], [3, Proposition 2.10]), and hence also from $C$ to $C$. Being both compact and linear, it is necessarily (Kantorovich and Akilov [9, p. 244]) completely continuous.

Defining another completely continuous operator $T: R \rightarrow C$ by

$$
T(w)(t):=f(t)+(K w)(t), \quad t \in[a, b], w \in R,
$$

and a continuous, bounded operator $G: C \rightarrow C$ by

$$
G(x)(t):=g(t, x(t)), \quad t \in[a, b], x \in C,
$$

we rewrite the integral equations (1.1) and (1.4) in operator form as

$$
y=T(\lambda G(y)), \quad y \in C,
$$

and

$$
z=\lambda G T(z), \quad z \in R,
$$

respectively. The two equations are equivalent in the sense of the following lemma.

LEMma 1 (Kumar and Sloan [13]). For $\lambda \in \mathbf{R}$, the sets

$$
\Theta_{T G}:=\{y \in C: T(\lambda G(y))=y\} \text { and } \Theta_{G T}:=\{z \in R: \lambda G T(z)=z\}
$$

are in one-to-one correspondence. Specifically, $\lambda G$ is a one-to-one operator from $\Theta_{T G}$ onto $\Theta_{G T}$, with inverse $T$.

Thus $z^{c}=\lambda^{c} G\left(y^{c}\right)$, and $y^{c}=T\left(z^{c}\right)$.

Note that since $T$ is completely continuous from $R$ to $C$, and $G$ is continuous and bounded on $C$, it follows (Krasnosel'skii and Zabreiko [10, p. 74]) that $T(\lambda G)$ is completely continuous on $C$, and $G T$ is completely continuous on $R$. The assumptions A3 and A4 ensure that $G$ is continuously Fréchet differentiable on $C$; its Fréchet derivative at $x \in C$ is the bounded linear operator $G^{\prime}(x)$ given by

$$
\left[G^{\prime}(x) w\right](t)=g_{v}(t, x(t)) w(t), \quad t \in[a, b], w \in C .
$$

If, in addition, $\mathrm{A} 1$ and $\mathrm{A} 2$ hold, then the operator $G T$ is continuously Fréchet differentiable on $R$; its Fréchet derivative at $z \in R$ is the completely continuous linear operator $(G T)^{\prime}(z)$ given by

$$
\left[(G T)^{\prime}(z) w\right](t)=G^{\prime}(T(z))(K w)(t), \quad t \in[a, b], w \in R .
$$


Furthermore, if A5 also holds, then the second Fréchet derivative of $G T$ at $z \in R$ is the symmetric bilinear operator $(G T)^{\prime \prime}(z)$, on $R \times R$ to $C$, given by

$$
\left[(G T)^{\prime \prime}(z) w x\right](t)=g_{v v}(t, T(z)(t))(K w)(t)(K x)(t), \quad t \in[a, b], w, x \in R .
$$

Similarly, $T(\lambda G)$ is twice Fréchet differentiable; its first and second Fréchet derivatives at $y \in C$ are given by

$$
\left[(T \lambda G)^{\prime}(y) w\right](t)=\lambda K G^{\prime}(y) w(t), \quad t \in[a, b], w \in C,
$$

and

$$
\left[(T \lambda G)^{\prime \prime}(y) w x\right](t)=\lambda K g_{v v}(t, y(t)) w(t) x(t), \quad t \in[a, b], w, x \in C,
$$

respectively.

The final assumption (A6) implies that for all $y_{1}, y_{2} \in B_{1}\left(y^{c}, \rho\right)$,

$$
\begin{aligned}
& \left\|(T \lambda G)^{\prime \prime}\left(y_{1}\right)-(T \lambda G)^{\prime \prime}\left(y_{2}\right)\right\| \\
& \quad \leq|\lambda| \sup _{a \leq t \leq b} \int_{a}^{b}|k(t, s)|\left|g_{v v}\left(s, y_{1}(s)\right)-g_{v v}\left(s, y_{2}(s)\right)\right| d s \\
& \quad \leq \alpha|\lambda| \sup _{a \leq t \leq b} \int_{a}^{b}|k(t, s)|\left|y_{1}(s)-y_{2}(s)\right| d s \\
& \quad \leq \alpha|\lambda|\|K\|\left\|y_{1}-y_{2}\right\|_{\infty}
\end{aligned}
$$

where

$$
\|K\|=\sup _{a \leq t \leq b} \int_{a}^{b}|k(t, s)| d s<\infty .
$$

Likewise, for all $z_{1}, z_{2} \in B_{2}\left(z^{c}, \rho\right)$, where

$$
B_{2}\left(z^{c}, \rho\right)=\left\{z \in R:\left\|z-z^{c}\right\|_{\infty} \leq \rho /\|K\|\right\}
$$

it is easily verified that $T\left(z_{1}\right), T\left(z_{2}\right) \in B_{1}\left(y^{c}, \rho\right)$, and therefore

$$
\begin{aligned}
\left\|(G T)^{\prime \prime}\left(z_{1}\right)-(G T)^{\prime \prime}\left(z_{2}\right)\right\| \\
\quad \leq\|K\|^{2} \sup _{a \leq t \leq b}\left|g_{v v}\left(t, T\left(z_{1}\right)(t)\right)-g_{v v}\left(t, T\left(z_{2}\right)(t)\right)\right| \\
\quad \leq \alpha\|K\|^{2} \sup _{a \leq t \leq b}\left|T\left(z_{1}\right)(t)-T\left(z_{2}\right)(t)\right| \\
\quad \leq \alpha\|K\|^{3}\left\|z_{1}-z_{2}\right\|_{\infty} .
\end{aligned}
$$

3. The Approximate Method and its Convergence. Since $\left(z^{c}, \lambda^{c}\right)$ is a simple turning point of (2.2), the following hold by definition (see, for example, Moore and Spence [14], [15]):

D1. $z^{c}-\lambda^{c} G T\left(z^{c}\right)=0$

D2. $I-\lambda^{c}(G T)^{\prime}\left(z^{c}\right)$ has a one-dimensional null space spanned by $\phi^{c}$;

D3. $I-\lambda^{c}(G T)^{\prime}\left(z^{c}\right)$ has closed range;

D4. $\left[I-\lambda^{c}(G T)^{\prime}\left(z^{c}\right)\right]^{*}$ has a one-dimensional null space spanned by the linear functional $\sigma^{c}$;

(Here ${ }^{*}$ denotes the conjugate of a bounded linear operator on $R$.)

D5. $\sigma^{c}\left(G T\left(z^{c}\right)\right)=\sigma^{c}\left(z^{c}\right) / \lambda^{c} \neq 0$;

D6. $\sigma^{c}\left(\left[(G T)^{\prime \prime}\left(z^{c}\right)\right] \phi^{c} \phi^{c}\right) \neq 0$. 
It follows from properties D1 and D2 that $\left(z^{c}, \lambda^{c}\right)$ is a nonisolated solution of (2.2). However, $\left(z^{c}, \lambda^{c}\right)$ is contained as part of an isolated solution of the following system (Moore and Spence [14]):

$$
\begin{gathered}
z-\lambda G T(z)=0, \\
\phi-\lambda\left[(G T)^{\prime}(z)\right] \phi=0, \\
l(\phi)-1=0,
\end{gathered}
$$

where $\lambda \in \mathbf{R}, z, \phi \in R$ and $l$ is a bounded linear functional which normalizes $\phi$.

For our convergence analysis we rearrange (3.1) as

$$
\begin{gathered}
z-\lambda G T(z)=0, \\
\phi-\lambda\left[(G T)^{\prime}(\lambda G T(z))\right] \phi=0, \\
\lambda-[\lambda-l(\phi)+1]=0 .
\end{gathered}
$$

Note that (3.1) and $\left(3.1^{\prime}\right)$ are equivalent in the sense that any solution of (3.1) is a solution of $\left(3.1^{\prime}\right)$ and vice versa.

We now write $\left(3.1^{\prime}\right)$ in abbreviated form as

$$
Z-F(Z)=0
$$

where $Z=(z, \phi, \lambda)$, and $F$ is an operator on the Banach space $R \times R \times \mathbf{R}$. We equip this product space with the product norm

$$
\|Z\|=\max \left(\|z\|_{\infty},\|\phi\|_{\infty},|\lambda|\right), \quad Z \in R \times R \times \mathbf{R} .
$$

LEMMA 2 (Moore and Spence [14]). Suppose A1 to A5 hold, and $l\left(\phi^{c}\right)=1$. Then $Z^{c}=\left(z^{c}, \phi^{c}, \lambda^{c}\right)$ is an isolated solution of (3.2).

3.1. Algorithmic Details of the Approximation Procedure. In the present work we approximate $\left(z^{c}, \phi^{c}, \lambda^{c}\right)$ by $\left(z_{n}, \phi_{n}, \lambda_{n}\right)$, where $z_{n}$ and $\phi_{n}$ are of the form

$$
\begin{array}{ll}
z_{n}(t)=\sum_{j=1}^{n} a_{n j} u_{n j}(t), & t \in[a, b], \\
\phi_{n}(t)=\sum_{j=1}^{n} b_{n j} u_{n j}(t), & t \in[a, b],
\end{array}
$$

and $\left\{u_{n 1}, \ldots, u_{n n}\right\}$ is a set of basis functions for some chosen approximating space. We determine the unknowns $a_{n 1}, \ldots, a_{n n}, b_{n 1}, \ldots, b_{n n}$, and $\lambda_{n}$ by collocating (3.1) at $n$ distinct points $\tau_{n 1}, \ldots, \tau_{n n}$ in $[a, b]$ :

$$
\left.\begin{array}{c}
z_{n}\left(\tau_{n i}\right)-\lambda_{n}\left[G T\left(z_{n}\right)\right]\left(\tau_{n i}\right)=0 \\
\left(\tau_{n i}\right)-\lambda_{n}\left[(G T)^{\prime}\left(z_{n}\right) \phi_{n}\right]\left(\tau_{n i}\right)=0
\end{array}\right\}, \quad i=1, \ldots, n,
$$

Thus we discretize (3.1) by the standerd collocation method. 
In practice, the computation of the $2 n+1$ unknowns $a_{n 1}, \ldots, a_{n n}, b_{n 1}, \ldots, b_{n n}$, and $\lambda_{n}$ may be arranged in the following manner. Let

$$
\begin{gathered}
x_{j}= \begin{cases}a_{n j}, & j=1, \ldots, n, \\
b_{n, j-n}, & j=n+1, \ldots, 2 n, \\
\lambda_{n}, & j=2 n+1,\end{cases} \\
f_{i}=f\left(\tau_{n i}\right), \\
U_{i j}=u_{n j}\left(\tau_{n i}\right), \quad 1 \leq i, \ldots, n,
\end{gathered}
$$

and

$$
K_{i j}=\int_{a}^{b} k\left(\tau_{n i}, s\right) u_{n j}(s) d s, \quad 1 \leq i, j \leq n .
$$

Then the unknowns $x_{1}, \ldots, x_{2 n+1}$ satisfy the system of equations

$$
\begin{aligned}
\sum_{j=1}^{n} x_{j} U_{i j}-x_{2 n+1} g\left(\tau_{n i}, f_{i}+\sum_{j=1}^{n} x_{j} K_{i j}\right) & =0 \\
\sum_{j=1}^{n} x_{n+j} U_{i j}-x_{2 n+1} g_{v}\left(\tau_{n i}, f_{i}+\sum_{j=1}^{n} x_{j} K_{i j}\right)\left[\sum_{j=1}^{n} x_{n+j} K_{i j}\right]=0 & \\
l\left(\sum_{j=1}^{n} x_{n+j} u_{n j}\right)-1=1, \ldots, n, & ,
\end{aligned}
$$

Since $y^{c}=T\left(z^{c}\right)$, we define our approximation to $y^{c}$ to be

$$
y_{n}:=T\left(z_{n}\right)
$$

It then follows that

$$
y_{n}(t)=f(t)+\sum_{j=1}^{n} x_{j} \int_{a}^{b} k(t, s) u_{n j}(s) d s, \quad t \in[a, b] .
$$

3.2. Analysis of the Approximate Method. We consider the case where the collocation approximations $z_{n}$ and $\phi_{n}$ are sought in piecewise polynomial function spaces.

For any natural number $N$, let

$$
\Pi_{N}: a=s_{1}<s_{2}<\cdots<s_{N}<s_{N+1}=b
$$

be a partition of $[a, b]$, and let $h=h(N)=\max _{1 \leq i \leq N}\left(s_{i+1}-s_{i}\right)$. We assume that $h \rightarrow 0$ as $N \rightarrow \infty$ and that the partition $\Pi_{N}$ is quasi-uniform, that is, there exists a constant $\varsigma$ such that, for all $N, h \leq \varsigma \min _{1 \leq i \leq N}\left(s_{i+1}-s_{i}\right)$.

With $r$ a positive integer and $\nu$ an integer satisfying $0 \leq \nu<r$, let $S_{r, N}^{\nu}$ denote the space of piecewise polynomial functions of order $r$ and continuity $\nu$. This terminology may be better understood by noting that a function $\omega \in S_{r, N}^{\nu}$ if and only if it is a polynomial of degree $\leq r-1$ on each subinterval $\left(s_{i}, s_{i+1}\right), 1 \leq i \leq N$, and has $\nu-1$ continuous derivatives on $(a, b)$. If $\nu=0$, there is no continuity requirement at the breakpoints $s_{i}, 1 \leq i \leq N+1$, in which case we arbitrarily take each $\omega \in S_{r, N}^{0}$ to be right-continuous at $s_{1}=a$, and left-continuous at every other $s_{i}, 2 \leq i \leq N+1$. 
For $n=\operatorname{dim}\left(S_{r, N}^{\nu}\right)=(N-1)(r-\nu)+r$, let $\left\{u_{n j}\right\}_{j=1}^{n}$ be a set of basis functions for $S_{r, N}^{\nu}$, and choose a set $\left\{\tau_{n i}\right\}_{i=1}^{n}$ of distinct points in $[a, b]$ such that the $n \times n$ matrix $\left(u_{n j}\left(\tau_{n i}\right)\right)$ is nonsingular. Furthermore, let $P_{n}$ be the interpolatory projection operator from $C+S_{r, N}^{\nu}$ onto $S_{r, N}^{\nu}$, which satisfies the condition

$$
\left(P_{n} w\right)\left(\tau_{n i}\right)=w\left(\tau_{n i}\right), \quad i=1, \ldots, n,
$$

for all $w \in C+S_{r, N}^{\nu}$. Then our discretization of (3.1) is represented by the system of equations

$$
\begin{gathered}
z_{n}-\lambda_{n} P_{n} G T\left(z_{n}\right)=0, \\
\phi_{n}-\lambda_{n} P_{n} G^{\prime}\left(T\left(z_{n}\right)\right) K \phi_{n}=0, \\
l\left(\phi_{n}\right)-1=0
\end{gathered}
$$

where $z_{n}=P_{n} z_{n} \in S_{r, N}^{\nu}, \phi_{n}=P_{n} \phi_{n} \in S_{r, N}^{\nu}$, and $\lambda_{n} \in \mathbf{R}$.

While we use (3.3) for computational purposes, to prove the existence and convergence of a turning point of (3.3a), we consider the following discretization of $\left(3.1^{\prime}\right)$ :

$$
\begin{gathered}
z_{n}-\lambda_{n} P_{n} G T\left(z_{n}\right)=0, \\
\phi_{n}-\lambda_{n}\left[\left(P_{n} G T\right)^{\prime}\left(\lambda_{n} P_{n} G T\left(z_{n}\right)\right)\right] \phi_{n}=0, \\
\lambda_{n}-\left[\lambda_{n}-l\left(\phi_{n}\right)+1\right]=0 .
\end{gathered}
$$

This system we write in abbreviated form as

$$
Z_{n}-F_{n}\left(Z_{n}\right)=0
$$

where $Z_{n}=\left(z_{n}, \phi_{n}, \lambda_{n}\right)$, and $F_{n}$ is an operator from $R \times R \times \mathbf{R}$ to $S_{r, N}^{\nu} \times S_{r, N}^{\nu} \times \mathbf{R}$.

Compact operator equations of the form (3.2) and (3.4) have been analyzed by Spence and Moore [16]. Here we apply their third theorem to prove the convergence of a solution $Z_{n}$ of (3.4) to the solution $Z^{c}$ of (3.2). But before we can do this, we need to impose the additional constraints on the (collocation) points $\left\{\tau_{n i}\right\}_{i=1}^{n}$ that they be chosen to ensure that $P_{n}$ is uniformly bounded as an operator from $C+S_{r, N}^{\nu}$ to $S_{r, N}^{\nu}$, that is

$$
\left\|P_{n}\right\| \leq c_{3}
$$

where $c_{3}>0$ is independent of $n$, and

$$
\lim _{n \rightarrow \infty}\left\|w-P_{n} w\right\|_{\infty}=0 \quad \text { for all } w \in C .
$$

Remark 1. Throughout this paper, $c_{1}, \ldots, c_{5}$ denote positive generic constants which may take different values at their different occurrences but which are always independent of $n$. 
THEOREM 1. Let $\left(y^{c}, \lambda^{c}\right)$ be a simple turning point of $(2.1)$, and let $\left(z^{c}, \lambda^{c}\right)$ be the corresponding simple turning point of (2.2). Suppose A1 to A6 hold, the interpolatory operator $P_{n}$ satisfies (3.5) and (3.6), and $l\left(\phi^{c}\right)=1$. Then, for sufficiently large $n,\left(z_{n}, \phi_{n}, \lambda_{n}\right) \in S_{r, N}^{\nu} \times S_{r, N}^{\nu} \times \mathbf{R}$ is an isolated solution of system $\left(3.3^{\prime}\right)$, and

$$
\begin{aligned}
& \max \left(\left\|z^{c}-z_{n}\right\|_{\infty},\left\|\phi^{c}-\phi_{n}\right\|_{\infty},\left|\lambda^{c}-\lambda_{n}\right|\right) \\
& \leq c_{1}\left\|z^{c}-P_{n} z^{c}\right\|_{\infty}+c_{2}\left\|\phi^{c}-P_{n} \phi^{c}\right\|_{\infty} .
\end{aligned}
$$

Proof. An application of Theorem 3 of Spence and Moore [16] yields the result. Two of the less trivial assumptions to check are the collective compactness (Anselone [3, p. 94]) of $\left\{P_{n} G T, n \geq 1\right\}$ on $R$, and the pointwise convergence of $P_{n} G T$ to $G T$ on $R$. The former condition follows from Weiss [19], and the latter from the mapping properties of $G T$, and (3.6).

From Theorem 3 of Spence and Moore [16] we have immediately that

$$
\begin{aligned}
& \max \left(\left\|z^{c}-z_{n}\right\|_{\infty},\left\|\phi^{c}-\phi_{n}\right\|_{\infty},\left|\lambda^{c}-\lambda_{n}\right|\right) \\
& \quad \leq c_{5} \max \left(\left\|z^{c}-P_{n} z^{c}\right\|_{\infty},\left\|\phi^{c}-\lambda^{c}\left[\left(P_{n} G T\right)^{\prime}\left(P_{n} z^{c}\right)\right] \phi^{c}\right\|_{\infty}\right) .
\end{aligned}
$$

The result now follows from the argument

$$
\begin{aligned}
\| \phi^{c}- & \lambda^{c}\left[\left(P_{n} G T\right)^{\prime}\left(P_{n} z^{c}\right)\right] \phi^{c} \|_{\infty} \\
\leq & \left\|\phi^{c}-\lambda^{c}\left[\left(P_{n} G T\right)^{\prime}\left(z^{c}\right)\right] \phi^{c}\right\|_{\infty} \\
& \quad+\left\|\lambda^{c}\left[\left(P_{n} G T\right)^{\prime}\left(z^{c}\right)\right] \phi^{c}-\lambda^{c}\left[\left(P_{n} G T\right)^{\prime}\left(P_{n} z^{c}\right)\right] \phi^{c}\right\|_{\infty} \\
= & \left\|\phi^{c}-P_{n} \phi^{c}\right\|_{\infty}+\left\|\lambda^{c} P_{n}\left[(G T)^{\prime}\left(z^{c}\right)-(G T)^{\prime}\left(P_{n} z^{c}\right)\right] \phi^{c}\right\|_{\infty} \\
\leq & \left\|\phi^{c}-P_{n} \phi^{c}\right\|_{\infty}+c_{4}\left\|(G T)^{\prime}\left(z^{c}\right)-(G T)^{\prime}\left(P_{n} z^{c}\right)\right\|_{\infty} \\
\leq & \left\|\phi^{c}-P_{n} \phi^{c}\right\|_{\infty}+c_{3}\left\|z^{c}-P_{n} z^{c}\right\|_{\infty},
\end{aligned}
$$

where in going from the second last step to the final step we have used a mean value theorem (Kantorovich and Akilov [9, p. 500]) since $(G T)^{\prime \prime}(z)$ exists and is bounded for all $z$ in a neighborhood of $z^{c}$.

Remark 2. The fact that $\left(3.3^{\prime}\right)$ has an isolated solution $\left(z_{n}, \phi_{n}, \lambda_{n}\right)$ implies that $\left(z_{n}, \dot{\lambda}_{n}\right)$ is a simple turning point of $(3.3 \mathrm{a})$.

Remark 3. Conditions (3.5) and (3.6) do not hold in the case of collocation with global polynomials, and thus a different approach needs to be adopted to prove the convergence of $Z_{n}$ to $Z^{c}$. For a possible approach see Kumar [12].

Since our approximation to $y^{c}$ is

$$
y_{n}:=T\left(z_{n}\right)
$$

where $z_{n} \in S_{r, N}^{\nu}$ is part of the isolated solution $\left(z_{n}, \phi_{n}, \lambda_{n}\right)$ of system $\left(3.3^{\prime}\right)$ (see Theorem 1), it follows from

$$
\left\|y^{c}-y_{n}\right\|_{\infty}=\left\|K\left(z^{c}-z_{n}\right)\right\|_{\infty} \leq\|K\|\left\|z^{c}-z_{n}\right\|_{\infty}
$$

that the rate of convergence of $y_{n}$ to $y^{c}$ is no worse than that of $z_{n}$ to $z^{c}$. In the next section we show that the convergence rate of $y_{n}$ to $y^{c}$ (and also that of $\lambda_{n}$ to $\lambda^{c}$ ) may be better than that of $z_{n}$ to $z^{c}$. 
4. Superconvergence Results. For $1 \leq p<\infty$, let $L_{p}=L_{p}(a, b)$ denote the Banach space of measurable, real-valued functions on $(a, b)$ which have integrable $p$ th power. The norm on this space is defined by

$$
\|w\|_{p}:=\left(\int_{a}^{b}|w(s)|^{p} d s\right)^{1 / p}, \quad w \in L_{p} .
$$

Let $L_{\infty}=L_{\infty}(a, b)$ denote the Banach space of essentially bounded real-valued functions on $(a, b)$. This space has the norm

$$
\|w\|_{\infty}=\operatorname{ess}_{a<t<b}|w(t)|, \quad w \in L_{\infty} .
$$

Finally, let $W_{p}^{m}=W_{p}^{m}(a, b)$, for $m$ a nonnegative integer and $1 \leq p \leq \infty$, be the Sobolev space of functions $w$ such that $w^{(i)} \in L_{p}$ for $i=0,1, \ldots, m$, where $w^{(i)}$ is the $i$ th distributional derivative of $w$. This space we equip with the norm

$$
\|w\|_{m, p}=\sum_{i=0}^{m}\left\|w^{(i)}\right\|_{p}
$$

The following corollary to Theorem 1 holds for any set of collocation points for which the operator $P_{n}$ satisfies (3.5) and (3.6), and may be obtained quite easily from an approximation-theoretic result in Graham, Joe and Sloan [5, Theorem 3.1].

COROLlaRY 1. If $z^{c} \in W_{\infty}^{\mu}, \mu \geq 1$, and $\phi^{c} \in W_{\infty}^{\delta}, \delta \geq 1$, then

$$
\max \left(\left\|z^{c}-z_{n}\right\|_{\infty},\left\|\phi^{c}-\phi_{n}\right\|_{\infty},\left|\lambda^{c}-\lambda_{n}\right|\right)=O\left(h^{\xi}\right)
$$

where $\xi=\min (\mu, \delta, r)$.

Up to this point, the analysis predicts a convergence rate of $O\left(h^{\xi}\right)$ for both $\lambda_{n}$ and $y_{n}$. In this section we carry the analysis further, and hence show that, under suitable conditions, both $y_{n}$ and $\lambda_{n}$ may converge at a rate $o\left(h^{\xi}\right)$.

We begin by noting that corresponding to the enlarged system (3.1) for $\left(z^{c}, \lambda^{c}\right)$ is an analogous system for $\left(y^{c}, \lambda^{c}\right)$ :

$$
\begin{gathered}
y-T(\lambda G(y))=0, \\
\psi-\lambda K G^{\prime}(y) \psi=0, \\
L(\psi)-1=0,
\end{gathered}
$$

where $\lambda \in \mathbf{R}, y, \psi \in C$ and $L$ is a bounded linear functional which normalizes $\psi$.

For our subsequent analysis we rearrange (4.1) as

$$
\begin{gathered}
y-T(\lambda G(y))=0, \\
\psi-\left[(T \lambda G)^{\prime}(T \lambda G(y))\right] \psi=0, \\
\lambda-[\lambda-L(\psi)+1]=0 .
\end{gathered}
$$

Note that (4.1) and $\left(4.1^{\prime}\right)$ are equivalent in the sense that any solution of (4.1) is a solution of $\left(4.1^{\prime}\right)$ and vice versa. 
We now write $\left(4.1^{\prime}\right)$ in abbreviated form as

$$
Y-H(Y)=0 \text {, }
$$

where $Y=(y, \psi, \lambda)$, and $H$ is an operator on the Banach space $C \times C \times \mathbf{R}$. We equip this product space with the product norm

$$
\|Y\|=\max \left(\|y\|_{\infty},\|\psi\|_{\infty},|\lambda|\right), \quad Y \in C \times C \times \mathbf{R} .
$$

LEMMA 3 (Moore and Spence [14]). Let $\left\{\psi^{c}\right\}, L\left(\psi^{c}\right)=1$, be a basis of the null space of the operator $I-\lambda^{c} K G^{\prime}\left(y^{c}\right)$, and suppose A1 to A5 hold. Then $Y^{c}=\left(y^{c}, \psi^{c}, \lambda^{c}\right)$ is an isolated solution of (4.2).

From Lemmas 1 to 3 it follows, if $z=z^{c}$ and $\lambda=\lambda^{c}$, that

$$
\phi=\lambda^{c} G^{\prime}\left(y^{c}\right) \psi^{c}
$$

is a solution of (3.1b). Similarly, if $y=y^{c}$ and $\lambda=\lambda^{c}$, then

$$
\psi=K \phi^{c}
$$

is a solution of (4.1b). Thus, just as we approximate $y^{c}=T\left(z^{c}\right)$ by $y_{n}=T\left(z_{n}\right)$ (see (3.7)), we may approximate $\psi^{c}$ by

$$
\psi_{n}=K \phi_{n}, \quad L\left(\psi_{n}\right)=1,
$$

where $\phi_{n} \in S_{r, N}^{\nu}$ is part of the isolated solution $\left(z_{n}, \phi_{n}, \lambda_{n}\right)$ of system (3.3') (see Theorem 1).

Using $\left(3.3^{\prime}\right)$, we write (3.7) and (4.4) as the system of equations

$$
\begin{gathered}
y_{n}-T\left(\lambda_{n} P_{n} G\left(y_{n}\right)\right)=0 \\
\psi_{n}-\left[\left(T \lambda_{n} P_{n} G\right)^{\prime}\left(T \lambda_{n} P_{n} G\left(y_{n}\right)\right)\right] \psi_{n}=0 \\
L\left(\psi_{n}\right)-1=0
\end{gathered}
$$

and then abbreviate (4.5) as

$$
Y_{n}-H_{n}\left(Y_{n}\right)=0,
$$

where $Y_{n}=\left(y_{n}, \psi_{n}, \lambda_{n}\right)$, and $H_{n}$ is an operator on $C \times C \times \mathbf{R}$. An application of Theorem 3 of Spence and Moore [16] now gives the following result.

THEOREM 2. Let $\left(y^{c}, \lambda^{c}\right)$ be a simple turning point of $(2.1)$, and let $\left(z^{c}, \lambda^{c}\right)$ be the corresponding simple turning point of (2.2). Suppose A1 to A6 hold, the interpolatory operator $P_{n}$ satisfies (3.5) and (3.6), and $l\left(\phi^{c}\right)=1$. Then, for sufficiently large $n,\left(y_{n}, \psi_{n}, \lambda_{n}\right) \in C \times C \times \mathbf{R}$ is an isolated solution of system (4.5), and

$$
\begin{aligned}
& \max \left(\left\|y^{c}-y_{n}\right\|_{\infty},\left\|\psi^{c}-\psi_{n}\right\|_{\infty},\left|\lambda^{c}-\lambda_{n}\right|\right) \\
& \quad \leq c_{3}\left\|K\left(z^{c}-P_{n} z^{c}\right)\right\|_{\infty}+c_{4}\left\|K\left(\phi^{c}-P_{n} \phi^{c}\right)\right\|_{\infty} .
\end{aligned}
$$

Proof. To derive (4.7), note that Theorem 3 of Spence and Moore [16] gives immediately that

$$
\begin{aligned}
& \max \left(\left\|y^{c}-y_{n}\right\|_{\infty},\left\|\psi^{c}-\psi_{n}\right\|_{\infty},\left|\lambda^{c}-\lambda_{n}\right|\right) \\
& \quad \leq c_{5} \max \left(\left\|K\left(z^{c}-P_{n} z^{c}\right)\right\|_{\infty},\left\|K \phi^{c}-\left[\left(T \lambda^{c} P_{n} G\right)^{\prime}\left(T P_{n} z^{c}\right)\right] \psi^{c}\right\|_{\infty}\right),
\end{aligned}
$$

and an argument similar to that given in the proof of Theorem 1 yields

$$
\begin{aligned}
& \left\|K \phi^{c}-\left[\left(T \lambda^{c} P_{n} G\right)^{\prime}\left(T P_{n} z^{c}\right)\right] \psi^{c}\right\|_{\infty} \\
& \quad \leq\left\|K\left(\phi^{c}-P_{n} \phi^{c}\right)\right\|_{\infty}+c_{2}\left\|K\left(z^{c}-P_{n} z^{c}\right)\right\|_{\infty} .
\end{aligned}
$$


Inequality (4.7) is the key to the derivation of our superconvergence results for $y_{n}$ and $\lambda_{n}$. The results given here are for the cases $\nu=0$ and $\nu=1$ only, and appear in Theorem 3. It should be noted that Theorem 3 holds only if the collocation points $\left\{\tau_{n j}\right\}_{j=1}^{n}$ are chosen, for each case $\nu=0$ and $\nu=1$, in the specific way indicated below.

Case $\nu=0$. Recall that the dimension of $S_{r, N}^{0}$ is $n=N r$, and hence $N r$ collocation points are needed. These should be selected as follows. Let $\zeta_{1}, \ldots, \zeta_{r}$ be the zeros of the $r$ th degree Legendre polynomial $\Phi_{r}(s), s \in[-1,1]$. (Note that these zeros are known as the Gauss-Legendre points.) Take as the collocation points the points $\varsigma_{1}, \ldots, \zeta_{r}$ shifted linearly to each subinterval $\left(s_{i}, s_{i+1}\right), 1 \leq i \leq N$ :

$$
\tau_{n,(i-1) r+j}=\left(s_{i}+s_{i+1}+\left(s_{i+1}-s_{i}\right) \zeta_{j}\right) / 2, \quad 1 \leq j \leq r, 1 \leq i \leq N .
$$

Case $\nu=1$. In this case the collocation approximations to $z^{c}$ and $\phi^{c}$ are sought in $S_{r, N}^{1}$, the space of continuous piecewise polynomial functions, and hence $r$ is necessarily $\geq 2$. The $n=N r-N+1$ collocation points should be taken to be the breakpoints $s_{i}, 1 \leq i \leq N+1$, plus the $r-2$ Lobatto points (that is, the zeros of the first derivative of $\left.\Phi_{r-1}(s), s \in[-1,1]\right)$ shifted linearly to each subinterval $\left(s_{i}, s_{i+1}\right), 1 \leq i \leq N$. Thus, if we let $\zeta_{r-1}=1$ and, fo $: r \geq 3$, we let $\varsigma_{1}, \ldots, \zeta_{r-2}$ be the $r-2$ Lobatto points, then the collocation points are

$$
\tau_{n,(i-1)(r-1)+j+1}=\left(s_{i}+s_{i+1}+\left(s_{i+1}-s_{i}\right) \zeta_{j}\right) / 2, \quad 1 \leq j \leq r-1,1 \leq i \leq N
$$

with $\tau_{n 1}=s_{1}=a$.

For the above sets of collocation points, the corresponding interpolatory operator $P_{n}$ satisfies (3.5) and (3.6) (Vainikko and Uba [18]), and $Y_{n}$ may exhibit the (super)convergence rate indicated in the next theorem. (There, $k_{t}(s):=k(t, s)$ for $t, s \in[a, b]$.

THEOREM 3. Let $\left(y^{c}, \lambda^{c}\right)$ be a simple turning point of $(2.1)$, and let $\left(z^{c}, \lambda^{c}\right)$ be the corresponding simple turning point of (2.2). Suppose A1 to A6 hold, and $l\left(\phi^{c}\right)=1$. If $z^{c} \in W_{1}^{\mu}, \mu \geq 1, \phi^{c} \in W_{1}^{\delta}, \delta \geq 1$, and $k_{t} \in W_{1}^{m}, 1 \leq m \leq r$, with $\left\|k_{t}\right\|_{m, 1}$ bounded independently of $t$, then for sufficiently large $n$,

$$
\max \left(\left\|y^{c}-y_{n}\right\|_{\infty},\left\|\psi^{c}-\psi_{n}\right\|_{\infty},\left|\lambda^{c}-\lambda_{n}\right|\right)=O\left(h^{\gamma_{\nu}}\right)
$$

where $\gamma_{\nu}=\min (m+r, \mu, \delta, 2 r-2 \nu), \nu=0$ or 1 .

Proof. The result is derived from inequality (4.7) by the arguments of Graham, Joe and Sloan [5] for the case $\nu=0$, and Joe [7] for the case $\nu=1$.

Thus, even though $z_{n}$ exhibits at most an $O\left(h^{r}\right)$ convergence rate, both $y_{n}$ and $\lambda_{n}$ may exhibit up to an $O\left(h^{2 r}\right)$ convergence rate in the case $\nu=0$, and up to an $O\left(h^{2 r-2}\right)$ convergence rate in the case $\nu=1$. Note that there is no superconvergence when continuous piecewise-linear functions $(\nu=1, r=2)$ are used.

5. A Numerical Example. In this section we compute the simple turning point of an integral equation reformulation of the nonlinear two-point boundary value problem

$$
y^{\prime \prime}(t)-\lambda \exp (y(t))=0, \quad t \in(0,1) ; \quad y(0)=y(1)=0, \quad \lambda \geq 0,
$$


which arises in the theory of spontaneous combustion of an infinite slab of exothermically reacting material (Gray and Wake [6], Thomas [17]). This problem is symmetric about $t=1 / 2$, and has solutions of the form

$$
y(t)=\ln (\beta)-2 \ln (\cosh ((2 t-1) \sqrt{\lambda \beta / 8})), \quad t \in[0,1],
$$

where $\beta$ satisfies the equation

$$
\beta-\cosh ^{2}(\sqrt{\lambda \beta / 8})=0 .
$$

A simple turning point exists at $\lambda^{c}=3.513830719125 \ldots$, and there are two solutions for $0<\lambda<\lambda^{c}$.

The problem may be reformulated as the integral equation

$$
y(t)=-\lambda \int_{0}^{1} k(t, s) \exp (y(s)) d s, \quad t \in[0,1]
$$

where

$$
k(t, s)= \begin{cases}-s(1-t), & s \leq t \\ -t(1-s), & s>t .\end{cases}
$$

The enlarged system that we solve for $\left(z^{c}, \phi^{c}, \lambda^{c}\right)$ is

$$
\begin{gathered}
z(t)-\lambda \exp \left(-\int_{0}^{1} k(t, s) z(s) d s\right)=0 \\
\phi(t)+\lambda \exp \left(-\int_{0}^{1} k(t, s) z(s) d s\right) \int_{0}^{1} k(t, s) \phi(s) d s=0 \\
\phi(1 / 2)-1=0 .
\end{gathered}
$$

Results for the case of piecewise constant functions $(\nu=0, r=1)$, equally spaced breakpoints, and a set of collocation points consisting of the midpoints of each subinterval, are displayed in Table 1 . The observed rates of convergence may be deduced from the columns headed EPOH which contain estimates of the power of $h$. Note that the observed rates support the theoretical predictions of Corollary $1(\xi=1)$ and Theorem $3\left(\gamma_{0}=2\right)$.

TABLE $1(\nu=0, r=1)$

\begin{tabular}{rllllll}
\hline$N$ & $\left\|z^{c}-z_{n}\right\|_{\infty}$ & $\mathrm{EPOH}$ & $\left\|y^{c}-y_{n}\right\|_{\infty}$ & $\mathrm{EPOH}$ & $\left|\lambda^{c}-\lambda_{n}\right|$ & $\mathrm{EPOH}$ \\
\hline 4 & $2.60 \mathrm{E} 00$ & 0.984 & $1.24 \mathrm{E}-2$ & 2.14 & $5.64 \mathrm{E}-2$ & 1.99 \\
8 & $1.31 \mathrm{E} 00$ & 0.994 & $2.83 \mathrm{E}-3$ & 2.03 & $1.42 \mathrm{E}-2$ & 2.00 \\
12 & $8.79 \mathrm{E}-1$ & 0.997 & $1.24 \mathrm{E}-3$ & 2.03 & $6.34 \mathrm{E}-3$ & 2.00 \\
16 & $6.60 \mathrm{E}-1$ & 0.998 & $6.91 \mathrm{E}-4$ & 2.05 & $3.57 \mathrm{E}-3$ & 2.00 \\
20 & $5.28 \mathrm{E}-1$ & 0.999 & $4.38 \mathrm{E}-4$ & 2.01 & $2.28 \mathrm{E}-3$ & 2.00 \\
24 & $4.40 \mathrm{E}-1$ & & $3.03 \mathrm{E}-4$ & & $1.59 \mathrm{E}-3$ & \\
\hline
\end{tabular}

Table 2 shows the results for the case of discontinuous piecewise linear functions $(\nu=0, r=2)$, equally spaced breakpoints, and a set of collocation points consisting of the two Gauss-Legendre points, $-1 / \sqrt{3}$ and $1 / \sqrt{3}$, shifted linearly to each subinterval. Note that the results suggest an $O\left(h^{4}\right)$ convergence rate for both $y_{n}$ 
and $\lambda_{n}$ whereas, because $k_{t}(s) \in W_{1}^{1}(0,1)$ but $k_{t}(s) \notin W_{1}^{2}(0,1)$, Theorem 3 predicts a rate of only $O\left(h^{3}\right)$. This discrepancy occurs because the Sobolev spaces used in this paper are of integral order only: in such a setting the prediction of Theorem 3 is the best possible. If, however, the analysis were to be carried out in the fractional derivative space setting used by Joe [8] in his study of the superconvergence phenomenon for (linear) second-kind Fredholm integral equations then, for this case, an $h^{4}$ order of convergence would be predicted for both $y_{n}$ and $\lambda_{n}$.

TABLE $2(\nu=0, r=2)$

\begin{tabular}{rllllll}
\hline$N$ & $\left\|z^{c}-z_{n}\right\|_{\infty}$ & $\mathrm{EPOH}$ & $\left\|y^{c}-y_{n}\right\|_{\infty}$ & $\mathrm{EPOH}$ & $\left|\lambda^{c}-\lambda_{n}\right|$ & $\mathrm{EPOH}$ \\
\hline 4 & $5.68 \mathrm{E}-1$ & 1.79 & $7.20 \mathrm{E}-4$ & 3.80 & $9.25 \mathrm{E}-5$ & 7.27 \\
8 & $1.64 \mathrm{E}-1$ & 1.93 & $5.16 \mathrm{E}-5$ & 3.89 & $5.99 \mathrm{E}-7$ & 1.98 \\
12 & $7.50 \mathrm{E}-2$ & 1.97 & $1.06 \mathrm{E}-5$ & 3.94 & $2.69 \mathrm{E}-7$ & 3.38 \\
16 & $4.26 \mathrm{E}-2$ & 1.98 & $3.42 \mathrm{E}-6$ & 3.97 & $1.02 \mathrm{E}-7$ & 3.67 \\
20 & $2.74 \mathrm{E}-2$ & 1.99 & $1.41 \mathrm{E}-6$ & 3.99 & $4.48 \mathrm{E}-8$ & 3.79 \\
24 & $1.91 \mathrm{E}-2$ & & $6.81 \mathrm{E}-7$ & & $2.25 \mathrm{E}-8$ & \\
\hline
\end{tabular}

Acknowledgement. The author is grateful to Professor I. Sloan for assistance in the development of this work.

School of Mathematics

University of New South Wales

Sydney, NSW 2033, Australia

1. P. M. ANSELONE, "Uniform approximation theory for integral equations with discontinuous kernels," SIAM J. Numer. Anal., v. 4, 1967, pp. 245-253.

2. P. M. ANSELONE, "Collectively compact approximations of integral operators with discontinuous kernels," J. Math. Anal. Appl., v. 22, 1968, pp. 582-590.

3. P. M. ANSElONe, Collectively Compact Operator Approximation Theory and Applications to Integral Equations, Prentice-Hall, Englewood Cliffs, N. J., 1971.

4. K. Atkinson, I. Graham \& I. SlOan, "Piecewise continuous collocation for integral equations," SIAM J. Numer. Anal., v. 20, 1983, pp. 172-186.

5. I. G. GRAhAM, S. JOE \& I. H. SlOAN, "Iterated Galerkin versus iterated collocation for integral equations of the second kind," IMA J. Numer. Anal., v. 5, 1985, pp. 355-369.

6. B. F. GRAY \& G. C. WAKE, "Criticality in the infinite slab and cylinder with surface heat sources," Combustion and Flame, v. 55, 1984, pp. 23-30.

7. S. JOE, "Collocation methods using piecewise polynomials for second kind integral equations," J. Comput. Appl. Math., v. 12 \& 13, 1985, pp. 391-400.

8. S. JOE, The Numerical Solution of Second Kind Fredholm Integral Equations, Ph. D. Thesis, University of New South Wales, Sydney, 1985.

9. L. V. Kantorovich \& G. P. AKIlov, Functional Analysis, Pergamon Press, Oxford, 1982.

10. M. A. KRASNOSEL'SkiI \& P. P. Zabreiko, Geometrical Methods of Nonlinear Analysis, Springer-Verlag, Berlin, 1984.

11. S. KUMAR, "Superconvergence of a collocation-type method for Hammerstein equations," IMA J. Numer. Anal., v. 7, 1987, pp. 313-225.

12. S. KUMAR, "The numerical solution of Hammerstein equations by a method based on polynomial collocation," 1987, submitted for publication.

13. S. KUMAR \& I. H. SlOAN, "A new collocation-type method for Hammerstein integral equations," Math. Comp., v. 48, 1987, pp. 585-593.

14. G. MOORE \& A. SPENCE, "The calculation of turning points of nonlinear equations," SIAM J. Numer. Anal., v. 17, 1980, pp. 567-576.

15. G. MOORE \& A. SPENCE, "The convergence of operator approximations at turning points," IMA J. Numer. Anal., v. 1, 1981, pp. 23-38. 
16. A. SPENCE \& G. MOORE, "A convergence analysis for turning points of nonlinear compact operator equations," in Numerical Treatment of Integral Equations, ISNM, vol. 53 (J. Albrecht and L. Collatz, eds.), Birkhäuser Verlag, Basel, 1980, pp. 203-212.

17. P. H. THOMAS, "On the thermal conduction equation for self-heating materials with surface cooling," Trans. Faraday Soc., v. 54, 1958, pp. 60-65.

18. G. VAINIKKO \& P. UBA, "A piecewise polynomial approximation to the solution of an integral equation with weakly singular kernel," J. Austral. Math. Soc. Ser. B, v. 22, 1981, pp. 431-438.

19. R. WEISS, "On the approximation of fixed points of nonlinear compact operators," SIAM J. Numer. Anal., v. 11, 1974, pp. 550-553. 